\title{
Ferrofluid thin films as optical gaussmeters proposed for field and magnetic moment sensing
}

\author{
SWAPNA S NAIR*, S RAJESH ${ }^{\dagger}$, V S ABRAHAM ${ }^{\dagger \dagger}$ and M R ANANTHARAMAN \\ Department of Physics, Cochin University of Science and Technology, Cochin 682 022, India \\ $\dagger$ Biophysics and Microscopy Group, Molecular Cytology, University of Amsterdam, \\ 1090 GB Amsterdam, The Netherlands \\ ${ }^{\dagger}$ School of Engineering and Sciences, International University of Bremen, 28759, Bremen, Germany
}

MS received 18 January 2008; revised 20 December 2010

\begin{abstract}
Ferrofluids belonging to the series, $\mathrm{Ni}_{x} \mathrm{Fe}_{1-x} \mathrm{Fe}_{2} \mathrm{O}_{4}$ and $\mathrm{Zn}_{x} \mathrm{Fe}_{1-x} \mathrm{Fe}_{2} \mathrm{O}_{4}$, were synthesized using cold co-precipitation. Liquid films of these ferrofluids were prepared by encapsulating the ferrofluids in between two optically smooth and ultrasonically cleaned glass plates. Magnetic field induced laser transmission through these ferrofluid films has been investigated. Magnetic field values can be calibrated in terms of output laser power in the low field region in which the variation is linear. This set up can be used as a cheap optical gaussmeter in the low field regime. Using the same set-up, the saturation magnetization of the sample used can also be calculated with a sample that is pre-characterized. Hence both magnetization of the sample, as well as applied magnetic field can be sensed and calculated with a precalibrated sample.
\end{abstract}

Keywords. Ferrofluids; optical gaussmeter; ferrites; magneto-optics; laser transmission.

\section{Introduction}

Ferrofluids are stable colloidal suspensions of nano-sized ferro or ferrimagnetic materials in a suitable carrier fluid. Surfactant-based ferrofluids find extensive applications due to their excellent stability against gravitational settling and agglomeration (Rosensweig 1985; Berkovsky et al 1993; Bhat 1998) in which a steric repulsion is provided by coating the ultrafine magnetic particles which are in the critical size limit of $100 \AA$. They have extensive industrial applications such as those in making rotary seals, pressure sensors and loud speakers (Bhat 1998). They have enormous medical and biological applications including their use in cancer therapy (Pradhan et al 2007; Prasad et al 2007).

Zinc ferrite belongs to the category of normal spinels, in which zinc has exclusive preference to occupy the tetrahedral $A$ site while nickel ferrite is an inverse spinel (Ni has octahedral preference). Their nanoparticles possess novel magnetic properties. There is a well-defined order in their magnetic ultra fine particles giving them a superparamagnetic behaviour (Kittel 1971; Smit and Wijn 1959; Diatmar and Ahlers 1999; Pfannes et al 2001).

The series, $\mathrm{Zn}_{x} \mathrm{Fe}_{1-x} \mathrm{Fe}_{2} \mathrm{O}_{4}$ and $\mathrm{Ni}_{x} \mathrm{Fe}_{1-x} \mathrm{Fe}_{2} \mathrm{O}_{4}$, with increasing ' $x$ ' content in the steps of $0 \cdot 1$ will give us a behaviour varying from that shown by the parent ferrite, $\mathrm{Fe}_{3} \mathrm{O}_{4}$, in a serial manner due to the increasing zinc/nickel

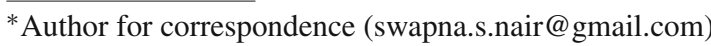

content. The ferrofluids prepared out of these powder precursors are also expected to follow a similar behaviour if the particle concentration is the same for all samples. Moreover, by the selection of the series, $\mathrm{Zn}_{x} \mathrm{Fe}_{1-x} \mathrm{Fe}_{2} \mathrm{O}_{4}$ and $\mathrm{Ni}_{x} \mathrm{Fe}_{1-x} \mathrm{Fe}_{2} \mathrm{O}_{4}$, the properties of a spinel ferrite as well as an inverse spinel ferrite can be studied at the nano level. Due to these peculiar properties, we selected them for the preparation of ferrofluids.

Optical properties of these smart fluids have been extensively studied. Linear and circular birefringence, dichroism and Faraday rotation are some among the magneto-optical properties shown by these rheological fluids which are investigated extensively (Davies and Llewellian 1980; Xu and Ridler 1997; Sutharia and Upadhyaya 1998; Horng et al 1999; Hasmonay and Depeyrot 2000; Bakuiz et al 2000a, b; Deperiot et al 2001; Pereira et al 2001; Wu et al 2001). However, a systematic study and correlation of the optical and magnetic properties shown by these fluids in device point of view has seldom been tried. In our present investigation, ferrofluids belonging to the series, $\mathrm{Zn}_{x} \mathrm{Fe}_{1-x} \mathrm{Fe}_{2} \mathrm{O}_{4}$ and $\mathrm{Ni}_{x} \mathrm{Fe}_{1-x} \mathrm{Fe}_{2} \mathrm{O}_{4}$, were synthesized and the optical and magnetic properties were correlated by a systematic study of the magnetic field-induced laser transmission through these ferrofluidic thin films. An optical gaussmeter can be formulated with these ferrofluid thin films with the help of an LDR, and a laser (a diode laser or a cheaper torch laser which gives a stream line light). This is a cheap optical gaussmeter set up in which the maximum magnetic field can be sensed and tuned by suitably choosing 
different ferrofluid film samples with varying saturation magnetization values. Using the same set up, the saturation magnetization of the sample used can also be calculated with a ferrofluid film that is precalibrated.

\section{Experimental}

\subsection{Preparation of magnetic fine particles}

Fine particles of $\mathrm{Ni}_{x} \mathrm{Fe}_{1-x} \mathrm{Fe}_{2} \mathrm{O}_{4}\left(\mathrm{Zn}_{x} \mathrm{Fe}_{1-x} \mathrm{Fe}_{2} \mathrm{O}_{4}\right)$ for the preparation of the ferrofluids were synthesized by the cold co-precipitation of the compounds $\mathrm{NiSO}_{4} \quad 7 \mathrm{H}_{2} \mathrm{O}\left(\mathrm{ZnSO}_{4}\right.$ $\left.7 \mathrm{H}_{2} \mathrm{O}\right), \mathrm{FeSO}_{4} 7 \mathrm{H}_{2} \mathrm{O}$ and $\mathrm{FeCl}_{3}$ in the molar ratio of $(1-x)$ $\mathrm{M}, x \mathrm{M}$ and $2 \mathrm{M}$ and coprecipitation is carried out in an alkaline medium with a $\mathrm{pH}$ around 10.5 ensuring the lowest possible particle size using $8 \mathrm{~N}$ ammonium hydroxide $\left(\mathrm{NH}_{4} \mathrm{OH}\right)$ and the precipitate is washed thoroughly with distilled water and finally with acetone to remove the excess $\mathrm{NH}_{4} \mathrm{OH}$. The obtained precipitate was dried and powdered.

\subsection{Reduction of particle size}

The particle size was reduced by high energy ball milling (HEBM). For the preparation of ultra fine particles, FRITSCH PULVERISETTE 7 PLANETARY MICRO MILL was employed. In this, 800 rotations per minute was achieved and hence the momentum imparted to the particles was very high. The ball to powder ratio was maintained at 1:8 to ensure a high momentum imparted to these fine particles. Therefore, the required size reduction was achieved within 2 or $3 \mathrm{~h}$. This results in excellent grinding performance at considerably shorter grinding times.

\subsection{Ferrofluid preparation}

Ferrofluids were prepared by milling the powder samples prepared by cold co-precipitation with the surfactant oleic acid, which provided the necessary steric repulsion by the polar ends of the molecule and thus preventing the agglomeration of the fine particles and finally milling the samples for $3 \mathrm{~h}$ with kerosene to have a uniform stable dispersion with a reduced particle size. The samples were centrifuged well to remove the sedimented particles and placed in an ultrasonic vibrator to enhance Brownian motion, which in turn provided stability to the ferrofluid. The ratio of oleic acid, kerosene and the fine particles of nickel iron ferrite were optimized.

\subsection{Ferrofluid thin film preparation}

The ferrofluid thin films belong to the series $\mathrm{Ni}_{x} \mathrm{Fe}_{1-x} \mathrm{Fe}_{2} \mathrm{O}_{4}$ $\left(\mathrm{Zn}_{x} \mathrm{Fe}_{1-x} \mathrm{Fe}_{2} \mathrm{O}_{4}\right)$ and were prepared by sandwiching and encapsulating $2 \mathrm{~mm}^{3}$ of ferrofluid in between two optically smooth and ultrasonically cleaned glass plates.

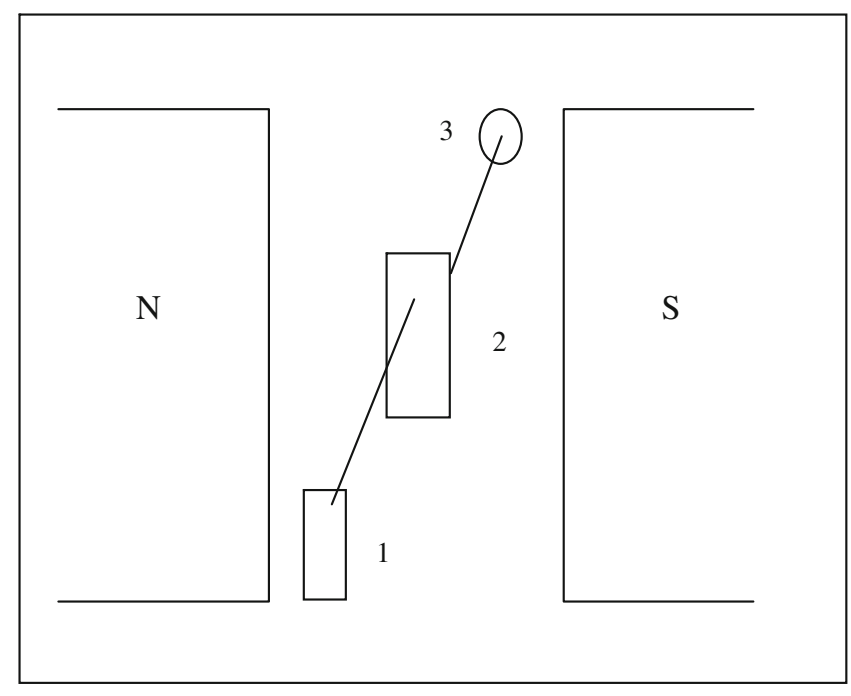

1.Laser 2. Detector 3. Sample

Figure 1. Construction of an optical Gaussmeter using ferrofluid films.

\subsection{Set up for optical gaussmeter}

The ferrofluid film sample was suspended in between the poles of a powerful electromagnet, which can go up to $1.5 \mathrm{~T}$ with water circulation for cooling the coil.

The film was suspended in such a way that it was always parallel to the magnetic field. A low power laser or searchlight, which had low beam divergence, was allowed to pass through the ferrofluid film samples normally. The transmitted output light was focussed on a light detector, which may be an LDR, and the resistance was measured in each applied magnetic field values (figure 1).

\section{Results and discussion}

As the ferrofluid liquid film restricts the motion of particles in a plane, application of a magnetic field increases structural anisotropy of these particles and agglomeration starts at a very low magnetic field and the particles self assemble themselves in the presence of applied magnetic field, yielding long chains and this process gets saturated at higher fields giving rise to a two-dimensional quasi-continuous grating. This is an example of field-induced assembling of nanomaterials. The chain formation saturates in higher fields giving thin long chains of varying thickness leading to optical anisotropy, which contributes to the optical birefringence.

At zero magnetic fields, surfactant coated ferrofluids show almost zero birefringence. Birefringence effects in any colloidal suspension may originate from the intrinsic optical anisotropy or the shape anisotropy of the fine magnetic particles. However, it is reported from electron microscopic observations that the shape anisotropy of the fine particles arising from the nonspherical nature of particles is very small in ferrofluids in comparison with the large optical 
anisotropy created in the chain formation in presence of the applied magnetic field. So we are getting almost zero birefringent signals in zero applied fields for these surfactant coated ferrofluids.

The optical properties of ferrofluids can be theoretically calculated by evaluating the orientational tensor $S_{\mathrm{ik}}=$ $(3 / 2)\left\{<n_{\mathrm{i}} n_{\mathrm{k}}>-1 / 3 \delta_{\mathrm{ik}}\right\}$ (Wu et al 2001). In the transverse magneto-optical effects, optical data is proportional to the square of the applied magnetic field as the eigenmodes are plane polarized waves. By processing the magneto-optical data at low field values, it can be found out that only the Zeeman energy term $\left(E_{\mathrm{z}}=-\mu H \cos \theta\right)$ is included in calculation of $<\cos ^{2} \theta>$ and so in the weak field approximation we get all the magneto-optical effects going linearly with $H^{2}$. Hence, in the magneto-transverse mode, a plot of optical transmission versus square of the magnetic field can yield a linear variation in low magnetic fields.

When light is transmitted through these ferrofluid films in the presence of the applied field, ultra fine magnetic particles suspended inside the medium will start to form tiny clusters which grow in size and assemble themselves along the magnetic field direction to form complete chains. Due to the chain formation the light gets scattered and the scattered light intensity increases with the applied field due to the increase in cluster formation. So as in the weak field approximation, we get almost a linear variation for output intensity with $H^{2}$. But as the cluster formation saturates in higher fields, output transmitted light intensity also tends to a constant value.

This phenomenon of the diffraction of light can be explained by treating the film in the presence of the field as a system of thin wires with variable thickness and spacing in between them due to the small magnetic field gradient away from the centre of the electromagnet. Then the different orders of the diffracted beam overlaps and we get a streak of light as we get diffracted from a thick wire.

Here, as there are a lot of thin wires of irregular thickness inside the film in the presence of a magnetic field, the situation is similar to the case of ' $\theta$ ' the angle of diffraction becomes very large and thus we get a continuous streak of light as in the case of a thin wire.

In the laser transmission studies, we have employed $3 \mathrm{~mW}$ laser (Diode laser-Red). Also the spot diameter on the ferrofluid film is of the order of 2-3 mm. Thus heating and drying up of the ferrofluid samples upon laser transmission can be minimized and so the output optical power is made time independent.

\subsection{Calibration of magnetic field in terms of optical power output}

It is observed that the diffracted light intensity increases with the field due to increase in the cluster formation. This in turn will reduce the central spot intensity. As the field increases, the central spot intensity decreases in a linear manner and then attains a constant value to the saturation of the cluster formation (Horng et al 2001). This is the saturation field $H_{\mathrm{s}}$ for that particular sample. After that the output intensity remains steady. Here the linear low field part in normalized power vs square of the applied field can be employed for magnetic field sensing for a particular sample. The normalized intensity vs applied magnetic field is plotted for the ferrofluid liquid thin films corresponding to $x=0 \cdot 1-0.6$ in steps of 0.1 in the series $\mathrm{Zn}_{x} \mathrm{Fe}_{1-x} \mathrm{Fe}_{2} \mathrm{O}_{4}$ and $\mathrm{Ni}_{x} \mathrm{Fe}_{1-x} \mathrm{Fe}_{2} \mathrm{O}_{4}$ and the representative figure is depicted as figure 2 . The exact dependence on the magnetic field is determined by curve fitting, which yields a value of $H^{2 \cdot 2}$ for all the samples.

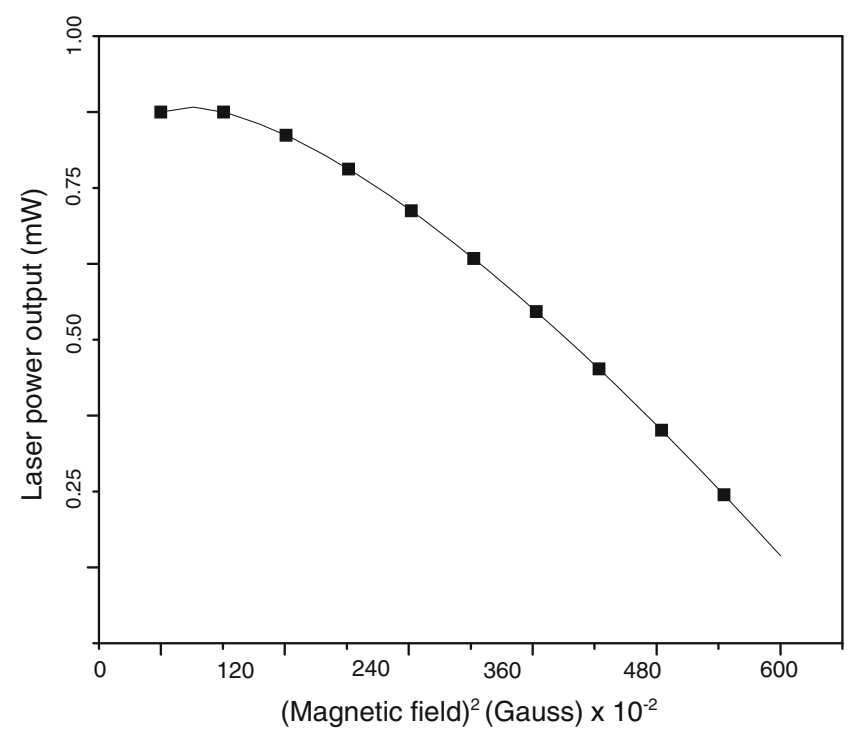

Figure 2. Magnetic field ${ }^{2}$ vs output power of $\mathrm{Ni}_{0 \cdot 1} \mathrm{Fe}_{0 \cdot 9} \mathrm{Fe}_{2} \mathrm{O}_{4}$.

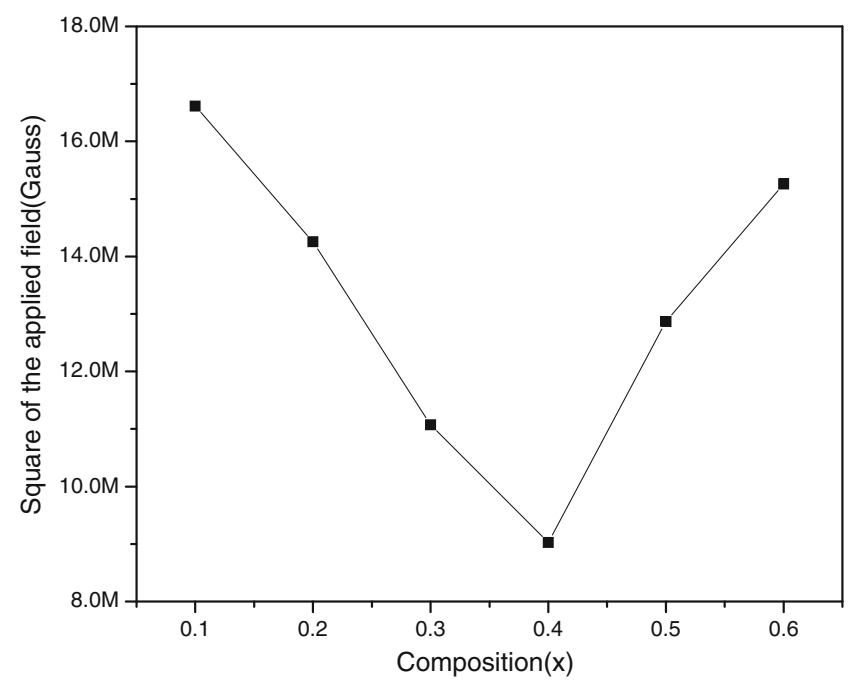

Figure 3. Composition vs saturating field ${ }^{2}$ in $\mathrm{Ni}_{x} \mathrm{Fe}_{1-x} \mathrm{Fe}_{2} \mathrm{O}_{4}$. 
The linear graph can be employed for the determination of the square of the magnetic field and thereby the magnetic field strength and the magnetic field can be thus calibrated in terms of optical output power. This set up is quite cheap with an LDR (Photodiode), a pointer laser and ferrofluid film. The most important application of this is in the magneto-optical applications where we have to simultaneously measure the magnetic field with the optical signal outputs normally. A more detailed investigation of the results have been provided in earlier publications (Abraham et al 2004; Swapna Nair et al 2006).

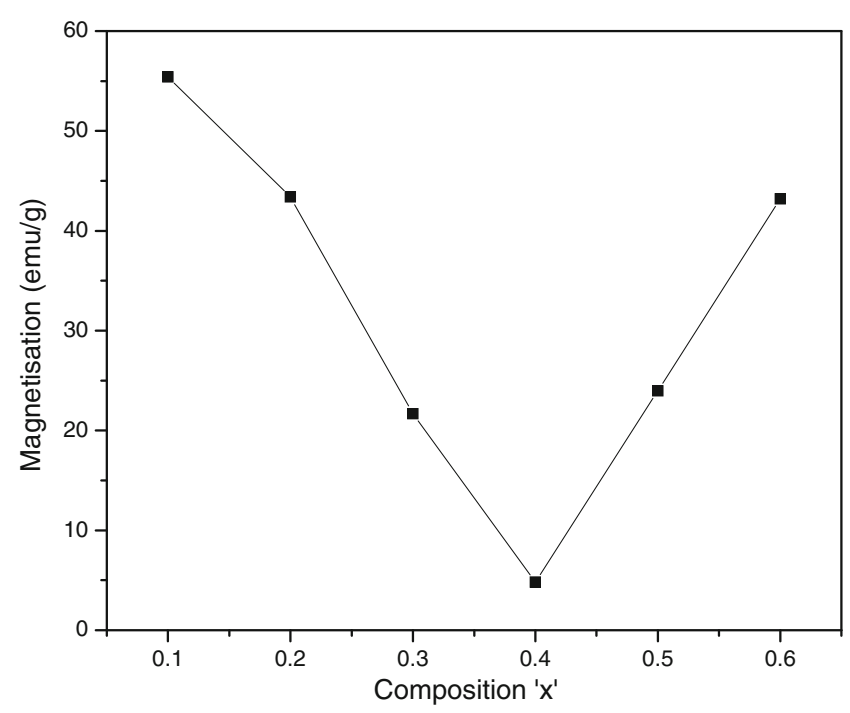

Figure 4. Composition vs saturation magnetization in $\mathrm{Ni}_{x} \mathrm{Fe}_{1-x} \mathrm{Fe}_{2} \mathrm{O}_{4}$.

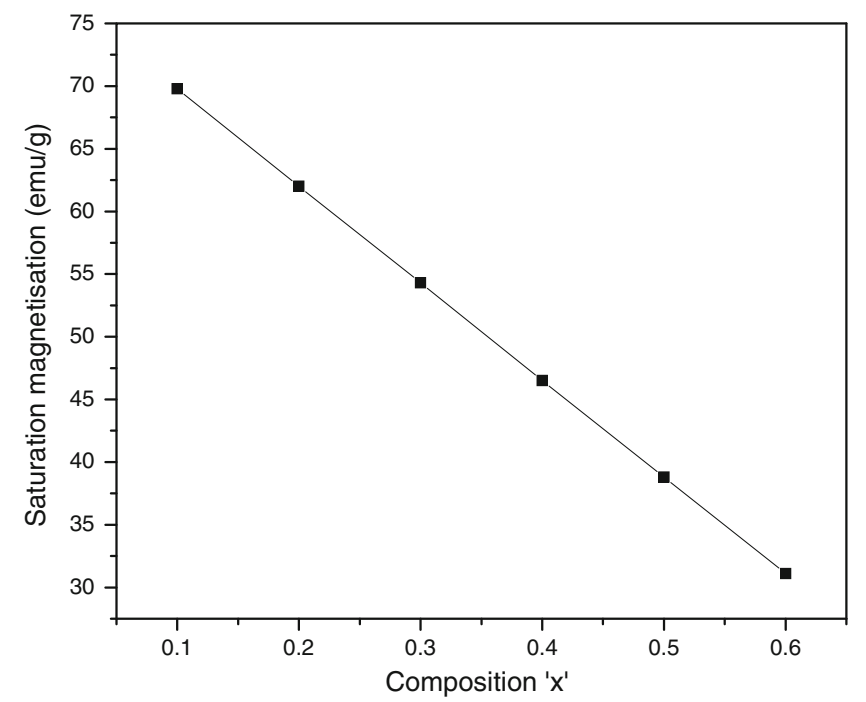

Figure 5. Composition vs saturation magnetization in $\mathrm{Zn}_{x} \mathrm{Fe}_{1-x} \mathrm{Fe}_{2} \mathrm{O}_{4}$.

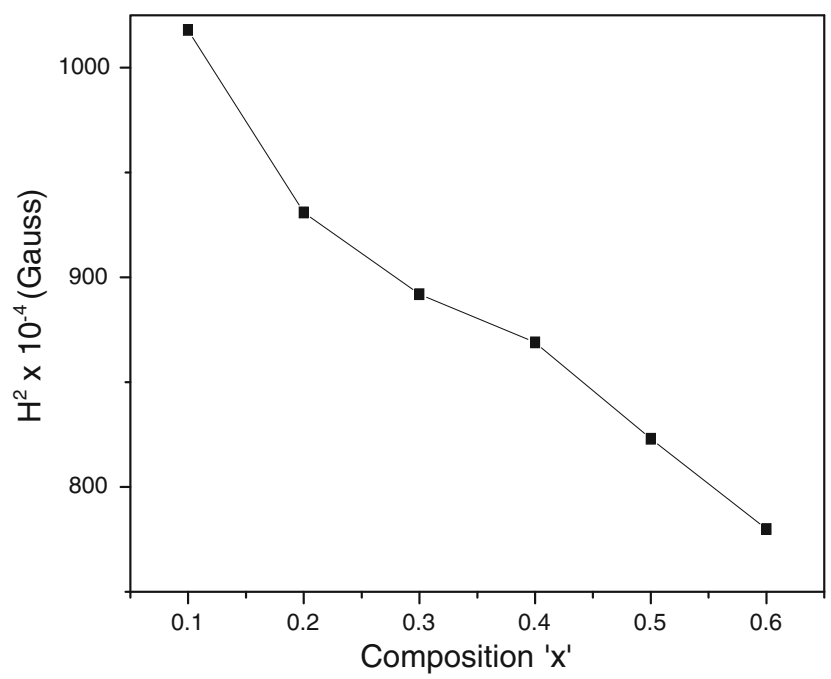

Figure 6. Composition vs saturation magnetization in $\mathrm{Zn}_{x} \mathrm{Fe}_{1-x} \mathrm{Fe}_{2} \mathrm{O}_{4}$.

\subsection{Calibration of saturation magnetization of samples}

All the magneto-optical effects are functions of their particle size and magnetization. Hence it is thought to calibrate the magneto-optical signals for any ferrofluid system in terms of a reference system whose magnetization is pre-characterized. Here in our system, ferrofluids belonging to the series $\mathrm{Ni}_{x} \mathrm{Fe}_{1-x} \mathrm{Fe}_{2} \mathrm{O}_{4}$ and $\mathrm{Zn}_{x} \mathrm{Fe}_{1-x} \mathrm{Fe}_{2} \mathrm{O}_{4}$ were investigated by the magnetic field assisted laser transmission studies for the correlation of their magnetic and magneto-optical transmission signal. Here it can be understood from figures 3 and 4 that the saturation value of magnetization is clearly a function of the saturation value for the optical signal for normalized particle volume. The same investigation is repeated for the $\mathrm{Zn}_{x} \mathrm{Fe}_{1-x} \mathrm{Fe}_{2} \mathrm{O}_{4}$ based ferrofluid thin film systems and a similar trend is observed (figures 5 and 6).

Hence the measurement of saturation magnetization/ magnetic moment (indirect) of the samples which can only be directly measured using sophisticated and costly techniques like vibrating sample magnetometry can be fructified. Also in ferrofluid-like system, the direct measurement of magnetization is still hectic a task due to its fluidic nature. However, this technique can be employed for the measurement of magnetization for a highly diluted system of fluids, which can even find immense practical applications.

\section{Conclusions}

We propose a device for the indirect measurement of magnetic moment using cheap optical techniques. For that, ferrofluids belonging to the series, $\mathrm{Ni}_{x} \mathrm{Fe}_{1-x} \mathrm{Fe}_{2} \mathrm{O}_{4}$ and $\mathrm{Zn}_{x} \mathrm{Fe}_{1-x} \mathrm{Fe}_{2} \mathrm{O}_{4}$, were investigated by the magnetic fieldassisted laser transmission studies for the correlation of their magnetic and magneto-optical transmission signal. The results correlate the magnetic moment and laser output, as 
well as magnetic field and laser output such that the ferrofluid film can be employed as a magnetic field and moment sensor. Thus using pre-characterized ferrofluid samples, the measurement of magnetic field and saturation magnetization can be done using simple cost-effective optical techniques however diluted the ferrofluid systems are. A device can be fabricated with fitted curves as the guidelines for the determination of the magnetic moment.

\section{References}

Abraham V S, Swapna Nair S, Rajesh S, Sajeev U S and Anantharaman M R 2004 Bull. Mater. Sci. 27165

Bakuiz A F, Da Silva M F, Morais P C and Skeff Neto K 2000a J. Appl. Phys. 872307

Bakuiz A F, Da Silva M F, Morais P C, Olavo L S F and Skeff Neto K 2000b J. Appl. Phys. 872497

Berkovsky B M, Medvedev V S and Krakov M S 1993 Magnetic fluids: Engineering applications (Oxford: Oxford University Press)

Bhat R K 1998 Indian J. Eng. Mater. Sci. 5477

Davies H W and Llewellian P 1980 J. Phys. D: Appl. Phys. 132327

Deperiot J, Da Silva G J and Alves C R 2001 Brazilian J. Phys. 31 390

Diatmar E and Ahlers H 1999 J. Magn. Magn. Mater. 192148
Hasmonay E and Depeyrot J 2000 J. Appl. Phys. 886628

Horng H E, Hong C Y, Yang H C, Jang I J, Yang S Y, Wu J M, Lee S I and Kuo F C 1999 J. Magn. Magn. Mater. 201215

Horng H E, Hong C Y, Yang S Y and Yang H C 2001 J. Phys. Chem. Solids 621749

Kittel C 1971 Introduction to solid state physics (New York: John Wiley) 4th edn

Pereira A R, Goncalves G R R, Bakuzis A F, Morais P C, Azevedo R B and Skeff Neto K 2001 IEEE Trans. Magn. 37 2657

Pfannes H D, Dias Filho J H, Magalhaes-Paniago R and Lopez J L 2001 Brazilian J. Phys. 31409

Pradhan P, Giri J, Samanta G, Sarma H D, Mishra K P, Bellare J, Banerjee R and Bahadur D 2007 J. Biomed. Mater. Res.: Part B-Appl. Biomater. B81 12

Prasad N K, Rathinasamy K, Panda D and Bahadur D 2007 J. Mater. Chem. 175042

Rosensweig R E 1985 Ferro hydrodynamics (Cambridge: Cambridge University Press)

Smit J and Wijn H P G 1959 Ferrites (Philips Technical Library)

Sutharia G M and Upadhyaya R V 1998 Indian J. Eng. Mater. Sci. 5347

Swapna Nair S, Rajesh S, Abraham V S and Anantharaman M R 2006 J. Magn. Magn. Mater. 30428

Wu K T, Kuo P C, Yao Y D and Tsai E H 2001 IEEE Trans. Magn. 372651

Xu M and Ridler P J 1997 J. Appl. Phys. 82326 